\title{
Adaptive Doppler centroid estimation algorithm of airborne SAR
}

\author{
Jian Yang ${ }^{1,2 a)}$, Chang Liu ${ }^{1}$, and Yanfei Wang ${ }^{1}$ \\ ${ }^{1}$ Institute of Electronics, Chinese Academy of Sciences \\ 19 North Sihuan Road, Haidian, Beijing 100190, China \\ ${ }^{2}$ Graduate University of Chinese Academy of Sciences \\ 10 South Ertiao Road, Haidian, Beijing 100039, China \\ a)yangjian.iecas@gmail.com
}

\begin{abstract}
Successfully imaging of airborne SAR requires that Doppler centroid frequency be accurately estimated. Energy balancing method is a classical method to solve this problem, whereas its performance relies heavily on the homogeneity of the scene. In this paper, the spectra of prominent point targets are analyzed, and then an adaptive algorithm based on Gaussian curve fitting is proposed to eliminate the impact of prominent point targets in the scene. Random estimation error is further eliminated by using linear fitting of estimations from each range cell. The results of simulations and the imaging of raw data prove the veracity of this algorithm.
\end{abstract}

Keywords: Doppler centroid estimation, energy balancing method, Gaussian curve fitting, synthetic aperture radar (SAR)

Classification: Microwave and millimeter wave devices, circuits, and systems

\section{References}

[1] F. K. Li, D. N. Held, J. C. Curlander, and C. Wu, "Doppler parameter estimation for spaceborne synthetic-aperture radars," IEEE Trans. Geosci. Remote Sens., vol. GE-23, no. 1, pp. 47-56, Jan. 1985.

[2] S. N. Madsen, "Estimating the Doppler centroid of SAR data," IEEE Trans. Aerosp. Electron. Syst., vol. 25, no. 2, pp. 134-140, March 1989.

[3] M. Y. Jin, "Optimal Doppler centroid estimation for SAR data from a quasi-homogeneous source," IEEE Trans. Geosci. Remote Sens., vol. GE24, no. 2, pp. 1022-1025, March 1986.

[4] R. Bamler, "Doppler frequency estimation and the Cramer-Rao bound," IEEE Trans. Geosci. Remote Sens., vol. 25, no. 3, pp. 385-390, May 1991.

[5] T. Long, Z. Lu, Z. Ding, and L. Liu, "A DBS Doppler centroid estimation algorithm based on entropy minimization," IEEE Trans. Geosci. Remote Sens., vol. 49, no. 10, pp. 3703-3712, Oct. 2011.

[6] S. Wei, Z. Fang, and H. Wang, "An improved method of Doppler centroid estimation in SAR," IGARSS 04', vol. 2, pp. 1530-1533, Sept. 2004.

[7] I. G. Cumming and F. H. Wong, Digital processing of synthetic aperture radar data: algorithms and implementation, Artech House, Norwood, 2005. 
[8] S. Engelberg, "The central limit theorem and low-pass filters," Proc. IEEE 2004 ICECS, pp. 65-68, Dec. 2004.

[9] H. Sun, H. Gu, W. Su, and G. Liu, "The study on Doppler centroid estimation in DBS imaging," Proc. 2001 CIE Int. Conf. Radar, pp. 679$682,2001$.

\section{Introduction}

Synthetic aperture radar (SAR) system plays an important role in geologic mapping and military surveillance, with its advantages of all-time, allweather, and high imaging resolution. Doppler centroid is an important parameter in imaging since inaccurate Doppler centroid results in signal-tonoise ratio (SNR) deterioration and image resolution degradation. However, in practical flight of airborne SAR, because of the imprecision of inertial navigation system (INS) data and motion error, the record of Doppler centroid departs from its actual value.

Algorithms have been presented to estimate Doppler centroid as an alternative to inaccurate INS data, such as energy balancing method [1], correlation Doppler estimator [2], sign Doppler estimator (SDE) [2], and maximumlikelihood estimation (MLE) [3]. The performances of these algorithms are compared in [4]. Energy balancing method, which is widely used in airborne SAR imaging, has a promising performance in homogeneous scenes, whereas its estimation precision declines sharply if the homogeneity is destroyed, especially when prominent point targets exist in the scene.

To solve this problem, an adaptive algorithm based on Gaussian curve fitting to eliminate prominent point targets is presented in this paper. By operating Gaussian curve fitting of the down-sampled azimuth power spectrum, the keen-edged and narrow power spectra of prominent point targets can be eliminated adaptively, and the homogeneity of power spectrum is improved. With linear fitting of estimations of each range cell, random estimation error can be further eliminated.

The paper is organized as follows. In Section 2, the principle of conventional algorithm is introduced and its limitations are analyzed. Section 3 presents the specific processing steps of the proposed algorithm. Section 4 shows the results of simulations and raw data imaging of both conventional and proposed algorithms. Finally, conclusive remarks are provided in Section 5 .

\section{Energy balancing method}

\subsection{Principle of energy balancing method}

The basis of energy balancing method is regarding that the amplitude of azimuth spectrum is generally the same as the pattern of antenna [5]. Thus, the energy center of azimuth spectrum can represent the estimation of Doppler centroid. 
The azimuth spectrum can be obtained by operating Fourier transform of azimuth data. Then, the initial value of Doppler centroid $f_{d c}$ can be calculated with INU data

$$
f_{d c}^{0}=\frac{2 v \cos \alpha \cos \varphi}{\lambda}
$$

where $v$ denotes the velocity of airplane, $\lambda$ represents radar wavelength, $\alpha$ is azimuth angle, and $\varphi$ is elevation angle. Iteratively change the value of $f_{d c}$ till the energy centroid of the spectrum is found, therefore the energy center is the Doppler centroid estimation.

However, if the scene is non-homogeneous, especially if prominent point targets exist in the scene, energy center cannot correctly replace Doppler centroid, and thus the energy balancing method is ineffective.

\subsection{Analysis of the homogeneity of scene}

In general, the average azimuth spectrum of SAR exhibits a pattern similar to the antenna power pattern in homogeneous scene [1]. However, in real azimuth spectrum, since prominent point targets exist in the scene, which have strong power, the homogeneity of the scene is destroyed, and the azimuth power spectrum will be asymmetrical.

Fig. 1 shows the azimuth power spectrum of real airborne SAR system in non-homogeneous scene. According to Fig. 1, there are mainly two kinds of prominent point targets that deteriorate the homogeneity of the scene. Target A represents targets with extremely high power that severely affects energy distribution of the spectrum, and Target B represents targets with considerable power, but relatively lower peak. Wei et al. [6] proposed an algorithm to remove the impact of Target A by setting up rigid threshold. However, the value of threshold is given manually, and the impact of Target $\mathrm{B}$ is neglected.

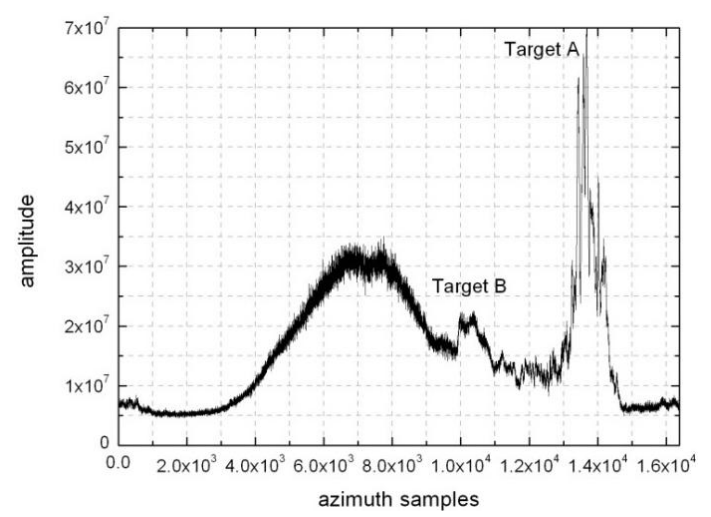

Fig. 1. Azimuth power spectrum of airborne SAR.

Moreover, according to signal detection theory, independent and identically distributed (IID) targets in the homogeneous scene can be modeled as Rayleigh distribution [7]. Since the echo can be regarded as the sum of targets in the scene, according to Central Limit Theorem [8], the SAR echo 
obeys Gaussian distribution. Therefore, its azimuth power spectrum is a Gaussian curve. It can be obtained from Fig. 1 that the spectra of prominent targets appear to be keen-edged and narrow, and the ideal azimuth power spectrum is a flat Gaussian curve. This feature of prominent point targets is the foundation of the proposed algorithm.

\section{Proposed algorithm}

According to the shapes of power spectra of prominent point targets, an improved energy balancing algorithm based on Gaussian curve fitting of down-sampled power spectrum is proposed. In this section, the principle and specific processing steps of the proposed algorithm are introduced.

Prominent point targets, such as Target A and B can be considered as disturbances of the Gaussian-shape azimuth spectrum. Moreover, their spectra are keen-edged and narrow, whereas the azimuth spectrum is relatively flat. After operate down-sample to the azimuth spectrum, only a few samples are located in Target A and B. On the contrary, most of the samples obey Gaussian distribution. Therefore, after Gaussian curve fitting, samples of ideal azimuth spectrum are preserved, and samples of prominent point targets will be abandoned adaptively.

The proposed algorithm consists of six steps as follows:

Step 1: Operating down-sample of the azimuth spectrum to get the discrete spectrum data;

Step 2: Operating Gaussian curve fitting of the discrete azimuth spectrum, thus samples of prominent point targets will be far from the fitting curve, which is defined as exceptional samples;

Step 3: Calculating the fitting errors of each sample, and calculate the mean and standard deviation of the fitting errors. Compare the fitting error of each sample with the sum of mean and standard deviation, the samples with fitting errors larger than the sum are regarded as exceptional samples and should be abandoned;

Step 4: Operating Gaussian curve fitting of the new spectrum without exceptive samples, the new fitting curve is considered to be the ideal azimuth power spectrum;

Step 5: Processing traditional energy balancing method to estimate Doppler centroid $\hat{f}_{d c}$

Step 6: Operating linear curving fitting of each range cell to further eliminate random estimation error [9].

The flowchart of the proposed algorithm is shown in Fig. 2. 


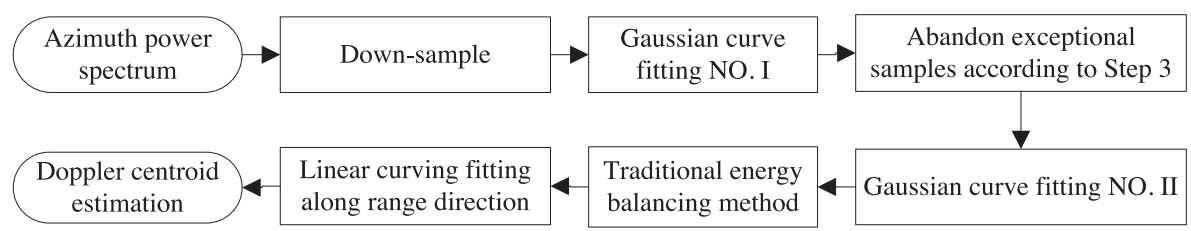

Fig. 2. Flowchart of the proposed algorithm.

\section{Simulations and raw data imaging}

In order to demonstrate the validity of the proposed algorithm, both simulation experiments and raw data are processed in this section.

In the simulation experiment, peak amplitude of the azimuth spectrum is set as 100, peak of Target A is 160, and peak of Target B is 40 . To better simulate the robustness of the proposed algorithm, white Gaussian noise is added to the spectrum with amplitude of 5 . Thus, the signal-to-noise ratio in this simulation is

$$
S N R=10 \log _{10} \frac{P_{\text {signal }}}{P_{\text {noise }}}=26.938 \mathrm{~dB}
$$

Fig. 3 (a) shows the simulated power spectrum of the azimuth data. Target $\mathrm{A}$ and $\mathrm{B}$ represent two kinds of prominent point targets analyzed in Section 2. From Fig. 3(b), the samples of Target A and B are far from fitting curve, and the peak of the fitting curve slightly departs from the actual Doppler center due to the impact of exceptional samples. Fig. 3 (c) shows that exceptional samples are abandoned, and new discrete azimuth spectrum is obtained. Fig. 3 (d) illustrates that after the second Gaussian curve fitting, the fitting curve is generally the same as the ideal azimuth power spectrum, and the center of the fitting curve is the same as the actual Doppler centroid. Therefore, the Doppler centroid can be estimated by operating traditional energy balancing algorithm. After processing the proposed algorithm, the homogeneity character of the scene is inherently improved, and the estimation precision is increased.

To testify the effectiveness of the proposed algorithm, imaging of real Ku-band airborne SAR data is processed. Fig. 3 (e) and 3 (f) show focusing performance before and after proposed algorithm without autofocus technique. It is clear that Fig. 3 (f) has a finer resolution, since Doppler centroid is estimated more precisely and RCM is completely corrected. Fig. 3 (g) offers the optical photo of the scene as a reference. Therefore, the validity of the proposed algorithm is confirmed.

\section{Conclusion}

This paper has proposed an adaptive algorithm based on Gaussian curve fitting to improve the performance of energy balancing method in non-homogeneous scene. First, the spectrum characters of the prominent point targets are analyzed in detail, and then Gaussian curve fitting is implemented to remove the impact of prominent point targets. Finally, random estimation error is 


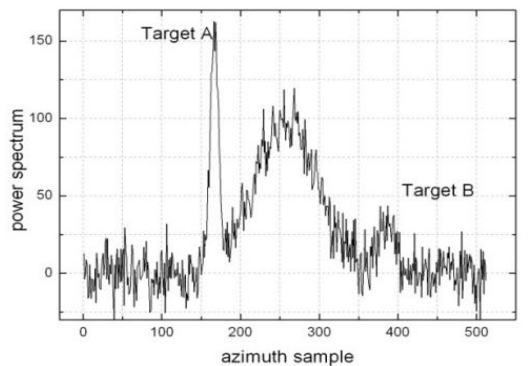

(a) Simulated azimuth spectrum

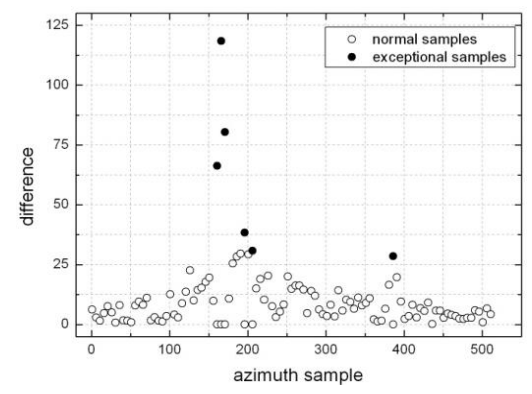

(c) Elimination of exceptional samples

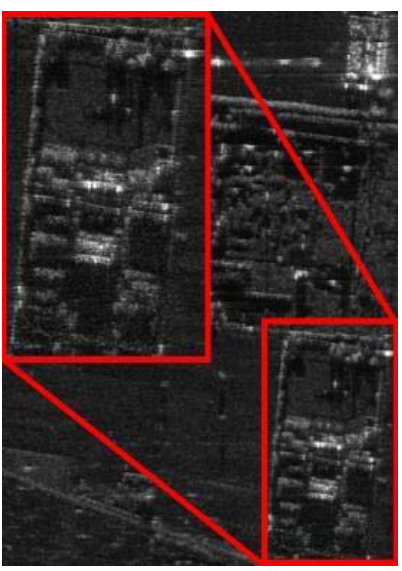

(e) Traditional algorithm

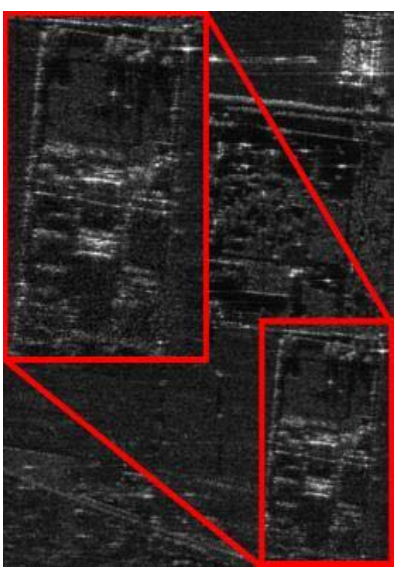

(f) Proposed algorithm

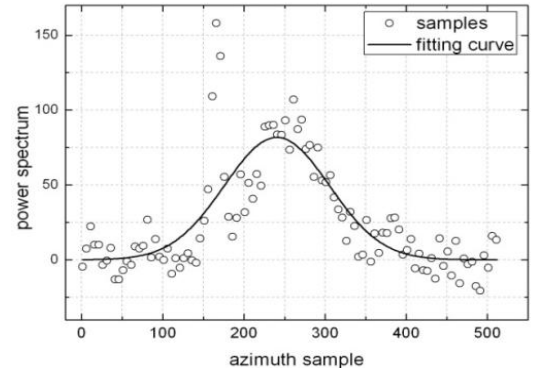

(b)After the first Gaussian curve fitting

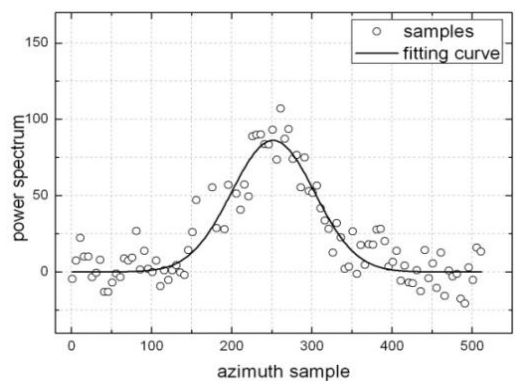

(d) After the second Gaussian curve fitting
Fig. 3. Results of simulations and raw data imaging.

further eliminated with linear fitting along range direction. The proposed algorithm has two advantages: 1) it inherently changes the homogeneity of the scene, which in turn improves the performance of energy balancing method in non-homogeneous scene; 2) it is an adaptive algorithm since both two kinds of targets are removed without setting up rigid threshold. The effectiveness of the proposed algorithm has been successfully validated through simulations and raw data imaging. 